\title{
Manganese Doped Fluorescent Paramagnetic Nanocrystals for Dual-Modal Imaging
}

\author{
Vijay Kumar Sharma, Sayim Gokyar, Yusuf Kelestemur, Talha Erdem, Emre Unal, \\ and Hilmi Volkan Demir*
}

\begin{abstract}
In this work, dual-modal (fluorescence and magnetic resonance) imaging capabilities of water-soluble, low-toxicity, monodisperse Mn-doped ZnSe nanocrystals (NCs) with a size $(6.5 \mathrm{~nm})$ below the optimum kidney cutoff limit $(10 \mathrm{~nm})$ are reported. Synthesizing Mn-doped ZnSe NCs with varying $\mathrm{Mn}^{2+}$ concentrations, a systematic investigation of the optical properties of these NCs by using photoluminescence (PL) and time resolved fluorescence are demonstrated. The elemental properties of these NCs using X-ray photoelectron spectroscopy and inductive coupled plasma-mass spectroscopy confirming $\mathrm{Mn}^{2+}$ doping is confined to the core of these NCs are also presented. It is observed that with increasing $\mathrm{Mn}^{2+}$ concentration the PL intensity first increases, reaching a maximum at $\mathrm{Mn}^{2+}$ concentration of 3.2 at\% (achieving a PL quantum yield (QY) of 37\%), after which it starts to decrease. Here, this high-efficiency sample is demonstrated for applications in dual-modal imaging. These NCs are further made water-soluble by ligand exchange using 3-mercaptopropionic acid, preserving their PL QY as high as 18\%. At the same time, these $N C s$ exhibit high relaxivity $\left(\approx 2.95 \mathrm{mM}^{-1} \mathrm{~s}^{-1}\right)$ to obtain $\mathrm{MR}$ contrast at $25^{\circ} \mathrm{C}, 3 \mathrm{~T}$. Therefore, the $\mathrm{Mn}^{2+}$ doping in these water-soluble Cd-free NCs are sufficient to produce contrast for both fluorescence and magnetic resonance imaging techniques.
\end{abstract}

\section{Introduction}

Colloidal semiconductor quantum dots (QDs), also known as nanocrystals (NCs) make an important class of inorganic fluorophores, which are gaining widespread recognition

\footnotetext{
Dr. V. K. Sharma, S. Gokyar, Y. Kelestemur, T. Erdem, E. Unal, Prof. H. V. Demir UNAM-Institute of Materials Science and Nanotechnology Department of Electrical and Electronics Engineering Department of Physics

Bilkent University

Ankara 06800, Turkey

E-mail: volkan@bilkent.edu.tr

Prof. H. V. Demir

Luminous! Center of Excellence for Semiconductor

Lighting and Displays

School of Electrical and Electronic Engineering

School of Mathematical and Physical Sciences

Nanyang Technological University

Singapore 639798, Singapore

DOI: $10.1002 / \mathrm{smll} .201401143$
}

because of their exceptional optical properties. These include high quantum yield (QY), broad absorption with narrow photoluminescence (PL) spectra, and a high resistance to photobleaching. This greatly enhances their potential of fluorescence-based imaging (FI)..$^{[1,2]}$ However, in bio-imaging, FI cannot provide three-dimensional (3D) anatomical information. In contrast, magnetic resonance imaging (MRI) is an important diagnostic tool with its ability to generate 3D images of opaque and soft tissues with sufficient spatial resolution and tissue contrast. ${ }^{[3,4]}$ Nevertheless, despite its imaging capability, the inherent low sensitivity of the MRI technique demands the synthesis of high relaxivity contrast enhancement agents. Contrast agents are currently applied in 30 to $40 \%$ of clinical MRI scans. Most of the commercial MR ( $T_{1}$ weighted) contrast agents contain the paramagnetic $\mathrm{Gd}^{3+}$ ion, which has seven unpaired electrons and a long electronic relaxation time. ${ }^{[5,6]}$ These contrast agents are intravenously administered to patients, reducing the relaxation time of water protons in the tissue of interest and increasing signal intensity. Recently, there have been efforts reported to combine different imaging techniques so that more information 
can be obtained from the sample under study. ${ }^{[7-9]}$ With two functionalities integrated into a single type of NCs, a sensitive contrast agent for two very powerful and highly complementary imaging techniques can be obtained. Therefore, multimodal imaging has stimulated intense interest for accurate medical diagnosis.

There are few reports in the literature on multimodal imaging based on the conjugation of QDs and magnetic nanoparticles (NPs). ${ }^{[10-16]}$ Most of these use cadmium $(\mathrm{Cd})$ based QDs, for example, $\mathrm{CdSe} / \mathrm{ZnS},{ }^{[10-14]} \mathrm{CdTe} / \mathrm{CdS},{ }^{[15]}$ $\mathrm{CdSeTe} / \mathrm{CdS}^{[16]}$ for FI. For MRI, $\mathrm{Gd}^{3+[10,11,16]}$ ion is commonly used for $T_{1}$ weighted imaging and $\mathrm{Fe}_{3} \mathrm{O}_{4}{ }^{[12-15]} \mathrm{NPs}$ for $\mathrm{T}_{2}$ weighted imaging in these previous reports. Cadmium is a toxic element, which limits its potential applications, especially related to human health. ${ }^{[17]}$ Although gadolinium $(\mathrm{Gd})$ has been the most popular choice among the paramagnetic metals, it has been recently linked to a medical condition known as nephrogenic systemic fibrosis (NSF). ${ }^{[18]}$ NSF is a rare but potentially harmful side effect observed in some patients with severe renal disease or following liver transplant. For obvious reasons, this has led to concerns over the safety of Gd-based $T_{1}$ contrast enhancement agents in MRI applications. ${ }^{[19,20]}$

Recently, Mn-doped NCs have been regarded as a promising new class of nanophosphors, owing to their superior luminescent properties and potential applications in optoelectronics ${ }^{[21]}$ and bio-imaging. ${ }^{[22]}$ They exhibit a broad emission peak at $585 \mathrm{~nm}$, with a large stoke's shift of $160 \mathrm{~nm}$, avoiding the issue of self-absorption. The $\mathrm{Mn}^{2+}(\mathrm{S}=5 / 2)$ is also used as a paramagnetic probe in several solids with a magnetic moment of $5 \mu_{\mathrm{B}}$. Manganese is considered to be safe for use in MRI contrast agents with no relation to NSF. The only issue is with the overexposure to free Mn ions, which must be avoided not to risk neurode-generative disorder. ${ }^{[18]}$

There are only two known reports ${ }^{[23,24]}$ of Mn-doped NCs used for dual-modal imaging. Wang et al. ${ }^{[23]}$ reported high QY $(\approx 21 \%$ in water $)$ and high relaxivity for $\mathrm{CdSe} / \mathrm{Zn}_{1-\mathrm{x}} \mathrm{Mn}_{\mathrm{x}} \mathrm{S}$ QDs. However, the problem with these QDs is the intrinsic toxicity of $\mathrm{Cd}$, which limits its widespread applications. Another issue is that, $\mathrm{Mn}$ is present in the shell, which is again potentially toxic if released from the shell. ${ }^{[18]}$ Recently, there is another report on dual imaging contrast agent by Gaceur et al. ${ }^{[24]}$ using Mn-doped ZnS NPs. This work reports blue-green emission in $\mathrm{Zn}_{0.9} \mathrm{Mn}_{0.1} \mathrm{~S}$ NPs with a high relaxivity $\approx 20 \mathrm{mM}^{-1} \mathrm{~s}^{-1}$, but the QY of these NPs has not been studied in the paper. This report used $\mathrm{Mn}^{2+}$ doping for the MRI imaging, but not for the FI. Different than the previous literature, here we are reporting smaller, water-soluble and low toxicity Mn-doped ZnSe NCs exhibiting high PL QY for FI and high relaxivity for MRI. These NCs are Cd-free and have $\mathrm{Mn}^{2+}$ doping mostly localized in the core of the NCs, which is confirmed by elemental characterization. Thus, our NCs can be considered to be less toxic with a sufficient $\mathrm{Mn}^{2+}$ concentration in the core region enabling both high QY and high contrast in MRI. Moreover, these NCs also meets another important criterion related to their size. They are $6.5 \mathrm{~nm}$ in size, well below the optimum kidney cutoff limit, which is $10 \mathrm{~nm}$. Therefore, these NCs can be used in most parts of the human body. ${ }^{[25]}$ Here, these NCs are unique in that, together

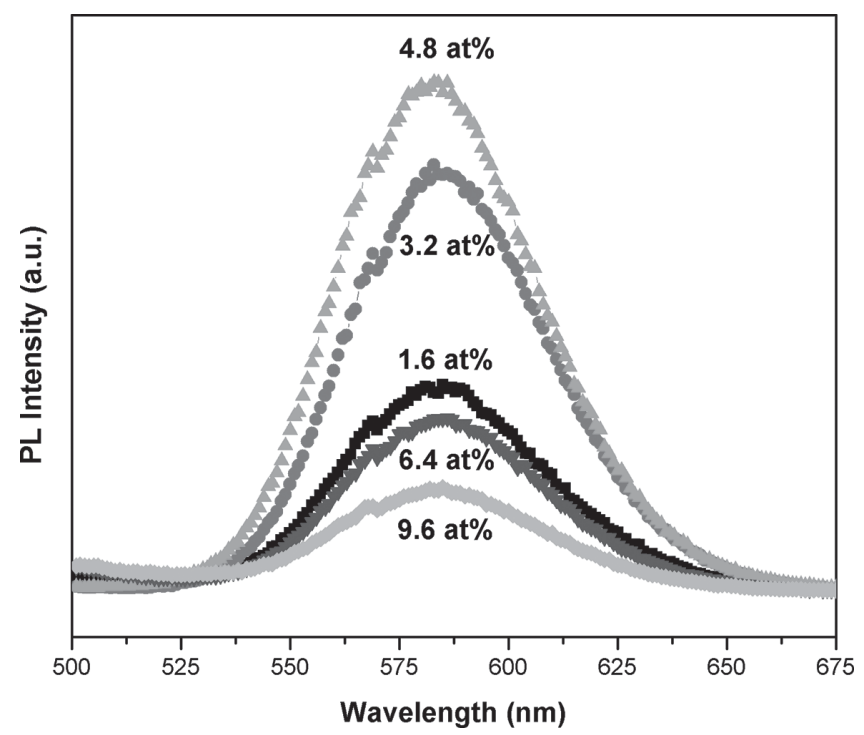

Figure 1. PL spectra of Mn-doped ZnSe NCs for different $\mathrm{Mn}^{2+}$ concentrations. The excitation wavelength used is $300 \mathrm{~nm}$.

with their small size $<<10 \mathrm{~nm}$ (below kidney cutoff limit) and $\mathrm{Mn}^{2+}$ doping confinement within the core, they offer high PL QY for FI and high relaxivity for MRI, while being water-soluble.

\section{Results and Discussion}

Mn-doped ZnSe NCs were synthesized using nucleation doping strategy ${ }^{[21]}$ which is explained in detail in the experimental section. The influence of $\mathrm{Mn}^{2+}$ doped concentration on the PL properties of Mn-doped ZnSe NCs was studied and presented in Figure 1. The injected $\mathrm{Mn}^{2+}$ concentration was varied from 1.6 to 10.8 at $\%$ to investigate the effect of $\mathrm{Mn}^{2+}$ on the optical properties of these NCs while keeping the other experimental conditions fixed (with a constant molar ratio $\left(\mathrm{Zn}(\mathrm{St})_{2} / \mathrm{TBPSe} / \mathrm{SA}=3.16 / 2.4 / 1.4\right)$. The dopant PL peak position was found to be independent of the $\mathrm{Mn}^{2+}$ concentration (with PL emission peaking at $585 \mathrm{~nm}$ and fullwidth-half-maximum $(\mathrm{FWHM}) \approx 54 \mathrm{~nm})$. It was observed that increasing the $\mathrm{Mn}^{2+}$ concentration from 1.6 to 4.8 at\%, increases the PL emission intensity, which indicates the successful incorporation of $\mathrm{Mn}^{2+}$ into $\mathrm{ZnSe} \mathrm{NCs}$. The PL QY gradually increases from 14 to $37 \%$ by increasing the $\mathrm{Mn}^{2+}$ concentration from 1.6 to 4.8 at $\%$.

Further increasing the $\mathrm{Mn}^{2+}$ concentration (above 4.8 at\%) leads to a decrease in the PL QY of these NCs. When the concentration of $\mathrm{Mn}^{2+}$ is higher than this threshold, the nonradiative energy transfer between neighboring $\mathrm{Mn}^{2+}$ dopant ions suppresses the fluorescence due to the concentration quenching effect. ${ }^{[26,27]}$ Under our synthesis conditions, the formation of such pairs of $\mathrm{Mn}^{2+}$ dopant ions is observed when the amount of $\mathrm{Mn}^{2+}$ ions in QDs is higher than 4.8 at\%. It is considered that the number of the luminescent $\mathrm{Mn}^{2+}$ ions increases within nanocrystals (MnSe core and/or MnSe/ZnSe interface) in the low concentration region. On the other hand, heavy doping with $\mathrm{Mn}^{2+}$ increases the nonradiative processes 


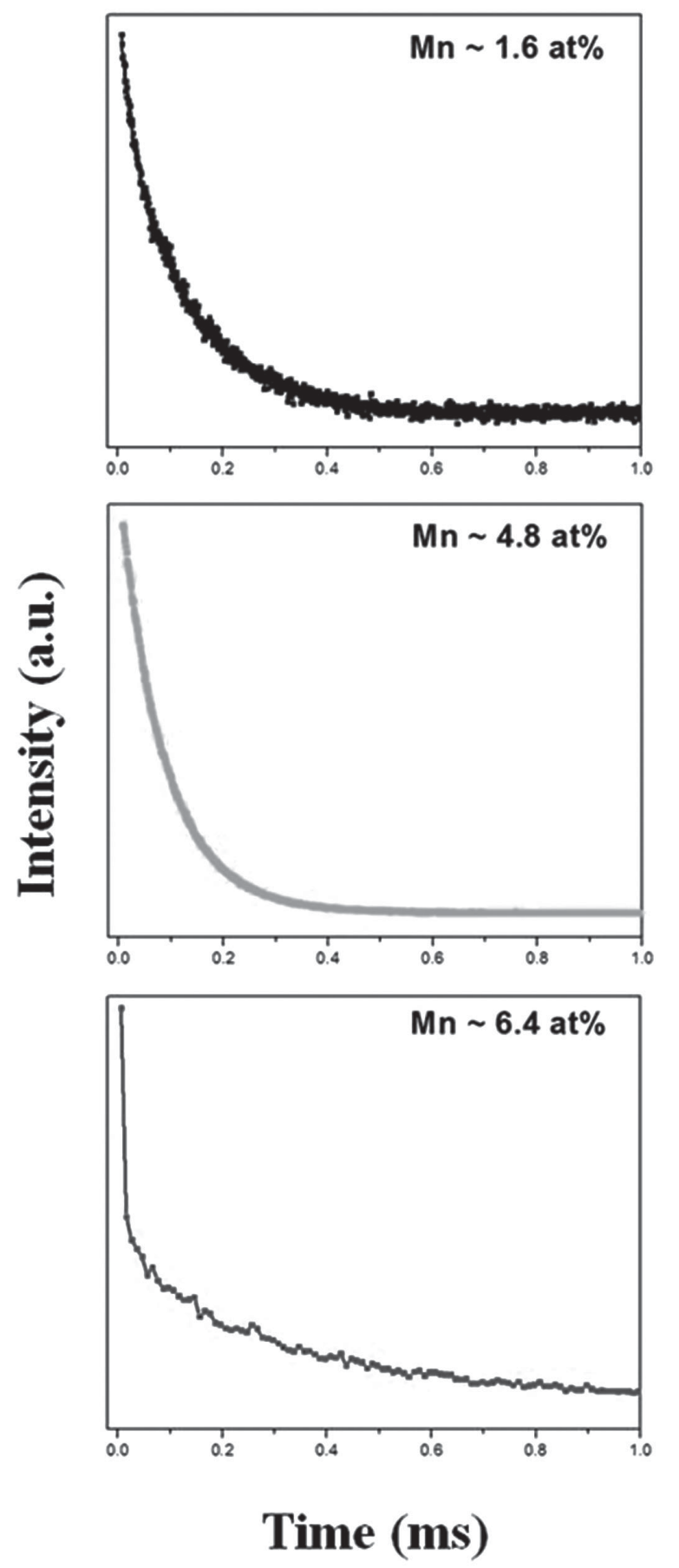

Figure 2. Time-resolved fluorescence decay profiles measured at $585 \mathrm{~nm}$ of Mn-doped ZnSe NCs at different $\mathrm{Mn}^{2+}$ concentrations under excitation of $310 \mathrm{~nm}$.

due to the formation of pairs of $\mathrm{Mn}^{2+}$ dopant ions, which in turn decreases the PL QY.

To obtain a better understanding of the effect of $\mathrm{Mn}^{2+}$ concentration on the emission kinetics, we performed timeresolved fluorescence (TRF) measurements on these NCs. Figure 2 shows the PL decay profiles of these NCs with varying $\mathrm{Mn}^{2+}$ concentrations excited at $310 \mathrm{~nm}$ and monitored at $585 \mathrm{~nm}$. The PL decay profiles exhibit an initial sharp spike due to the surface defects followed by the longer decay components measured in $\mathrm{ms}$ due to the $\mathrm{Mn}^{2+}$ ions. For the sample with $\mathrm{Mn}^{2+}$ concentration of 1.6 at $\%$, the decay profile of the $\mathrm{Mn}^{2+} \mathrm{PL}$ is found to be a single exponential with a lifetime of $0.1 \mathrm{~ms}$. As the $\mathrm{Mn}^{2+}$ concentration increases above 4.8 at \%, a fast decay component appears in addition to the $0.1 \mathrm{~ms}$ lifetime component. The PL decay of isolated $\mathrm{Mn}^{2+}$ ions is slow because the ${ }^{4} T_{1}-{ }^{6} A_{1}$ transition of single $\mathrm{Mn}^{2+}$ ions is parity- and spin-forbidden and is only weakly allowed through the odd parity crystal field and the spin-orbit interaction. It is known that the PL lifetime of $\mathrm{Mn}^{2+}$ pairs is shorter than that of single $\mathrm{Mn}^{2+}$ ions ${ }^{[28,29]}$ because in the exchange coupled $\mathrm{Mn}^{2+}$ pairs the spin selection rule is relaxed and the electric dipole transition is allowed. Therefore, we conclude that the faster decay PL component is attributed to the $\mathrm{Mn}-\mathrm{Mn}$ pairs in highly doped nanocrystals. This result is also supported by the observation of PL intensity decreasing with the increasing $\mathrm{Mn}^{2+}$ concentration above 4.8 at $\%$.

We also performed an elemental analysis of these NCs using X-ray photoelectron spectroscopy (XPS) and inductive coupled plasma-mass spectroscopy (ICP-MS) to determine the concentration of $\mathrm{Mn}^{2+}$ in these NCs. ICP-MS is one of the most sensitive and robust technique for measuring the elemental concentrations and XPS is selected for studying the core/shell nature of these NCs since it is a surface sensitive technique. ${ }^{[30]}$ The ratio of $\mathrm{Mn}^{2+}$ to $\mathrm{Zn}^{2+}$ in the Mn-doped ZnSe NCs determined by ICP-MS and XPS, compared with the corresponding injected values, are presented in Table $\mathbf{1}$. Since ICP-MS measurements provide the overall ratio of the two cations in a sample, the observed $\mathrm{Mn}^{2+}$ to $\mathrm{Zn}^{2+}$ ratio should reflect the real value. The Mn-to-Zn ratio of the NCs determined by XPS shows lower values, which is consistent with the targeted core/shell structure and suggests that $\mathrm{Mn}^{2+}$ ions are localized in the core. As a result, more $\mathrm{Zn}$ appears in the recording of XPS for the core/shell NCs. A core/shell structure with a diffused layer between the core and shell is also possible in our case as reported by Pradhan et al. ${ }^{[21]}$ for high synthesis temperature. From the above results, we observe that the sample with $\mathrm{Mn}^{2+}$ concentration $\approx 4.8$ at $\%$ (injected $\mathrm{Mn}^{2+}$ concentration) reaches the maximum PL QY $\approx 37 \%$. The actual value of $\mathrm{Mn}^{2+}$ concentration as confirmed by ICP-MS is 3.2 at $\%$.

We investigated Mn-doped ZnSe NCs with $\mathrm{Mn}^{2+}$ concentration $\approx 4.8$ at $\%$ having maximum PL QY as a potential contrast agent for FI and MRI. These NCs were rendered water-soluble by ligand exchange using 3-mercaptopropionic acid (MPA) for bio-medical applications. We studied and compared the optical and structural properties of these

Table 1. $\mathrm{Mn}^{2+}$ concentration (Mn/Zn molar ratio) obtained by ICP-MS and XPS for the Mn-doped ZnSe NCs.

\begin{tabular}{lcc}
\hline $\begin{array}{l}\text { Injected } \\
\text { [at\%] }\end{array}$ & $\begin{array}{c}\text { ICP-MS } \\
\text { [at\%] }\end{array}$ & $\begin{array}{c}\text { XPS } \\
\text { [at\%] }\end{array}$ \\
\hline 1.6 & 1.1 & 0.8 \\
3.2 & 1.8 & 1.3 \\
4.8 & 3.2 & 2.5 \\
6.4 & 4.4 & 3.6 \\
9.6 & 5.9 & 4.5 \\
\hline
\end{tabular}




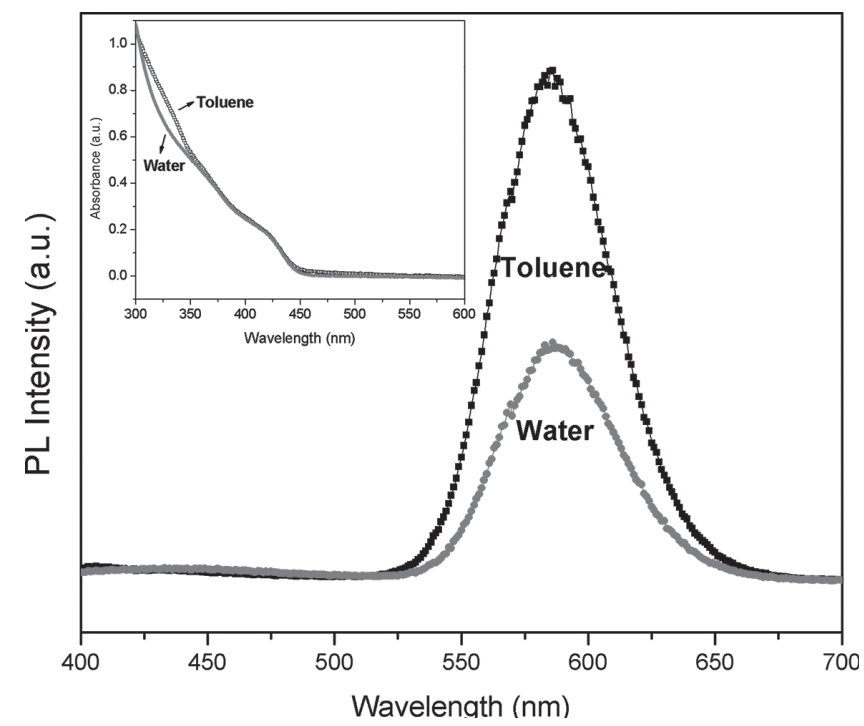

Figure 3. Photoluminescence and absorption (inset) spectra of toluene and water-soluble Mn-doped $\mathrm{ZnSe} \mathrm{NCs}$ with $\mathrm{Mn}^{2+}$ concentration (4.8 at\%). The excitation wavelength used is $300 \mathrm{~nm}$.

NCs is toluene and water. Figure 3 shows the PL spectra of the Mn-doped $\mathrm{ZnSe}\left(\mathrm{Mn}^{2+}\right.$ concentration $\approx 4.8$ at $\left.\%\right)$ NCs dissolved in toluene and water. The PL emission peak position is close in the two different solvents with similar FWHMs. Quantum yield for the water-soluble NCs $(18 \%)$ is reduced to almost half in comparison to toluene $(37 \%)$. The reduction of QY during the transfer from organic phase to water results from a combination of different effects. First, isolation of the NCs decreased the QY by $\approx 10-20 \%$ due to the loss of ligand molecules on the surface of the NCs and the further decrease of QY in water can be attributed to the dipole effect. These QY levels in water are comparable to the $\mathrm{CdSe} / \mathrm{Zn}_{1-\mathrm{x}} \mathrm{Mn}_{\mathrm{x}} \mathrm{S}$ QDs. ${ }^{[23]}$

In the UV-Vis absorption spectroscopy (shown in the inset of Figure 3) we observe the first excitonic peak at $425 \mathrm{~nm}$, which corresponds to the band edge absorption of the $\mathrm{ZnSe}$ NCs. While considering the Mn-dopant emission at $585 \mathrm{~nm}$, the Stoke's shift of $\approx 160 \mathrm{~nm}$ is observed as compared to the first excitonic absorption peak of the ZnSe NCs. Photoluminescence excitation (PLE) spectra (Figure S1 of Supporting Information) has similar features as the absorption spectra (excitonic peak $\approx 425 \mathrm{~nm}$ ) and the PL spectra (almost $50 \%$ drop in efficiency at $300 \mathrm{~nm}$ ). The PL decay profile (Figure S2 of Supporting Information) of the orange emission is found to be single exponential with an average lifetime of $0.1 \mathrm{~ms}$ for both toluene and water-soluble Mn-doped ZnSe NCs, which means the concentration of $\mathrm{Mn}^{2+}$ remains similar in both cases. This further confirms that the $\mathrm{Mn}^{2+}$ ions are only present in the core and not in the shell. Such slow decay further confirmed the assignment of this emission band to the spin forbidden doped $\mathrm{Mn}^{2+}{ }^{4} T_{1}$ to ${ }^{6} A_{1}$ transition. ${ }^{[31]}$

Transmission electron microscopy (TEM) was performed to understand the morphology and structure of these NCs. Figure 4a indicates that the size distribution of the Mn-doped ZnSe NCs was reasonably uniform with nearly spherical shape with an average diameter $\sim 6.5 \mathrm{~nm}$. Figure $4 \mathrm{~b}$ shows selected area electron diffraction (SAED) pattern and (high
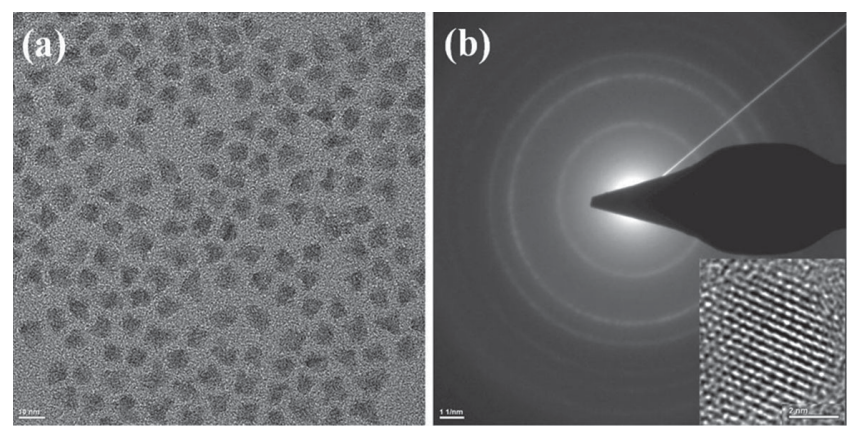

Figure 4. a) TEM and b) SAED of Mn-doped ZnSe NCs with $\mathrm{Mn}^{2+}$ concentration (4.8 at\%). In the inset, HR-TEM of Mn-doped ZnSe NCs is shown. Scale Bar is $10 \mathrm{~nm}$ for (a).

resolution-TEM) HR-TEM image of Mn-doped ZnSe NCs. SAED pattern shows three continuous rings with the interplanar spacing corresponding to the zinc blende structure of $\mathrm{ZnSe}$. The $d$ spacing of the rings from the center are; $d_{1} \approx$ $3.31 \AA, d_{2} \approx 2.03 \AA$, and $d_{3} \approx 1.73 \AA$ which can be indexed to (111), (220), and (311) planes of the zinc blende structure of ZnSe. HR-TEM image corresponds to $d$ spacing $\approx 3.33 \AA$ indexed to the most prominent (111) zinc blende phase of $\mathrm{ZnSe}$ (JCPDS 80-0021). It is also observed that, the average size and size distribution of the NCs remained same after the ligand exchange (not shown here).

The utility of the water-soluble Mn-doped ZnSe NCs as dual-mode imaging contrast agents was investigated in solution. NCs were studied by MRI and fluorescence microscopy to confirm that $\mathrm{Mn}^{2+}$ content was sufficient to produce contrast in MR image and also the QY is enough to produce fluorescence contrast in FI.

Particles were dissolved in water at concentrations of $0.01,0.02,0.045$, and $0.065 \mathrm{mM} \mathrm{Mn}^{2+}$ with DI water as a reference sample. $T_{1}$-weighted images for increasing concentration of $\mathrm{Mn}^{2+}$ with DI water as reference is shown in Figure 5. We can clearly observe the increase in the image contrast with the increase in $\mathrm{Mn}^{2+}$ concentration. Relaxation time $T_{1}$ was measured at $3 \mathrm{~T}, 25^{\circ} \mathrm{C}$ using a Spin Echo sequence. From the slope of $\left(1 / T_{1}-1 / T_{0}\right)$ vs $\mathrm{Mn}^{2+}$ concentration as shown in the Figure S3 of Supporting Information, we obtain the relaxivity $\left(r_{1}\right)$ value $\approx 2.95 \mathrm{mM}^{-1} \mathrm{~s}^{-1}$, where $T_{0}$ is relaxation time of DI water. Comparable relaxivity values were reported for $\mathrm{Gd}$

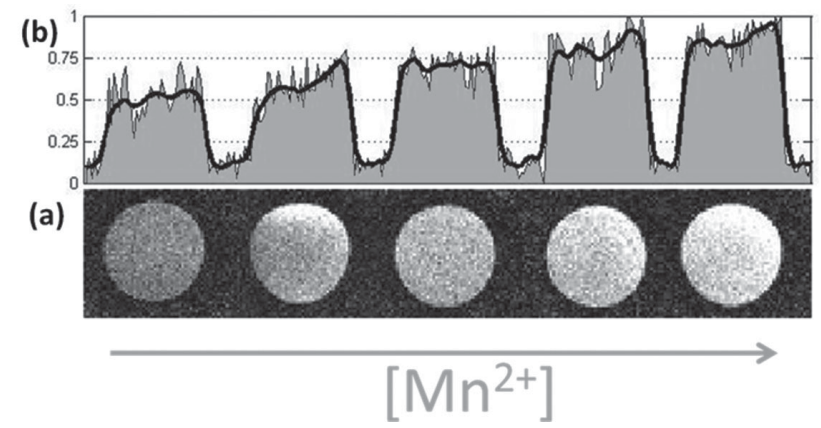

Figure 5. MRI of Mn-doped ZnSe NCs; a) from the Left (DI Water) to right (higher $\mathrm{Mn}^{2+}$ concentration). b) Integrated intensity of the same samples for clear understanding. The black line represents the average of these signals. 


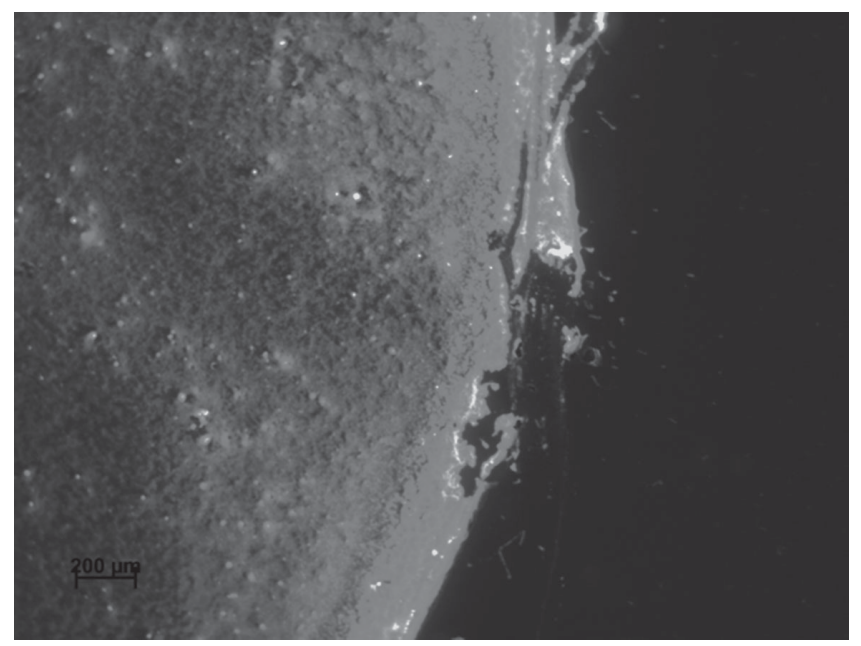

Figure 6. Fluorescence Image of a drop cast Mn-doped ZnSe NCs on quartz substrate.

based MR contrast agents and it is observed that the relaxivity increases with the increase in the values of magnetic field. ${ }^{[32]}$ Thus, these NCs offer a potential alternative to the Gd based MR contrast agents. In addition, our NCs possess high fluorescent QY which can be used as an additional tool for medical diagnosis along with MRI.

To demonstrate that these NCs possesses ample luminescence for optical imaging, samples of the same concentrations of NCs employed in the MR studies were imaged using fluorescence microscopy. NCs at a concentration that produced high signal enhancement for MRI were dropped on quartz and imaged by fluorescence microscopy with $405 \mathrm{~nm}$ diode laser excitation, $560 \mathrm{~nm}$ long pass filter at the detector. The high intensity of emission observed under these weak excitation conditions indicates the high QY of these NCs (Figure 6).

From the PLE spectra of Mn-doped emission $(\approx 585 \mathrm{~nm})$ (Figure S1, Supporting Information), we observe that PL emission is maximum for the excitation wavelength of $300 \mathrm{~nm}$, whereas the emission is only $50 \%$ of the maximum value at an exciation wavelength of $405 \mathrm{~nm} . \mathrm{Mn}^{2+}$ doped NCs also have a potential of multiphoton excitation which improves tissue penetration depth and resolution of in vivo. ${ }^{[22]}$ Thus, in our case we are reporting low toxic (Cd-free) NCs with high $\mathrm{QY}$ for fluorescence imaging with $\mathrm{Mn}$ atoms too localized in the core and not in the shell. Therefore, the doped $\mathrm{Mn}^{2+}$ concentration in these NCs is sufficient for both high QY for FI and high contrast in MRI.

\section{Conclusion}

In this work, we have demonstrated that Mn-doped ZnSe NCs are potential candidates for dual-modal imaging. They possess high QY (18\% in water) for fluorescence imaging and high relaxivity $\left(\approx 2.95 \mathrm{mM}^{-1} \mathrm{~s}^{-1}\right)$ for magnetic resonance imaging. These NCs are small in size $(\approx 6.5 \mathrm{~nm})$ and less toxic for use in the human body. Thus, these NCs hold a great promise for improved diagnosis using dual mode imaging.

\section{Experimental Section}

Materials: Zinc stearate ( $\mathrm{ZnSt}_{2}$, purum $10-12 \% \mathrm{Zn}$ basis), octadecylamine (ODA, 97\%), 1-octadecene (ODE, technical grade $90 \%$ ), tributyl phosphine (TBP, $\geq 93.5 \%$ ), stearic acid (SA, $\geq 98.5 \%$ ) and manganese chloride $\left(\mathrm{MnCl}_{2}, \geq 99 \%\right)$, were purchased from SigmaAldrich. Selenium powder (Se, $\geq 99.5 \%$ ), tetramethylammonium hydroxide (TMAH, $25 \mathrm{wt} \%$ in methanol), 3-mercaptopropionic acid (MPA, $\geq 99 \%$ ) and methanol (anhydrous, 99.8\%) was purchased from Alfa Aesar. All chemicals were used without further purification.

Synthesis of Manganese Stearate $\left(\mathrm{MnSt}_{2}\right)$ : In a typical synthesis, SA (2.25 g) was dissolved in $15 \mathrm{~mL}$ of anhydrous methanol and heated to $75^{\circ} \mathrm{C}$ until it became a clear solution. The solution of TMAH was prepared by taking $0.7 \mathrm{~mL}$ in $5 \mathrm{~mL}$ anhydrous methanol and mixed with SA solution. The mixture was stirred for $15 \mathrm{~min}$ in a beaker. To this solution, $\mathrm{MnCl}_{2}$ solution of $0.5 \mathrm{~g}$ in $5 \mathrm{~mL}$ anhydrous methanol was added drop wise with vigorous stirring and a white precipitate of $\mathrm{MnSt}_{2}$ slowlyflocculated. The synthesis is carried out inside glove box. The precipitates were washed repeatedly with hot methanol. Then the white precipitant was dried under vacuum for two days. The synthesized $\mathrm{MnSt}_{2}$ was stored inside glove box.

Preparation of Stock Solutions: TBPSe stock solution was prepared in a glove box by adding $1.9 \mathrm{~g}$ of Se into $10 \mathrm{~mL}$ of TBP by stirring at room temperature. The zinc stock solution was prepared by dissolving $\mathrm{ZnSt}_{2}(1.8 \mathrm{~g})$ and $0.4 \mathrm{~g}$ of SA in $10 \mathrm{~mL}$ of ODE. The selenium precursor solution was prepared by mixing $1 \mathrm{~mL}$ of TBPSe stock solution and $1 \mathrm{~g}$ of ODA in a vial heated at $75^{\circ} \mathrm{C}$ inside the glove box until it turns clear. Amine precursor solution was prepared by mixing $0.5 \mathrm{~g}$ of ODA and $0.63 \mathrm{~mL}$ of ODE in a vial at $75^{\circ} \mathrm{C}$ inside glove box.

Synthesis of Mn-doped ZnSe NCs: In a typical reaction, variable amount of $\mathrm{MnSt}_{2}(0.05,0.10,0.15,0.20$ and $0.30 \mathrm{~g})$ and $25 \mathrm{~mL}$ of ODE was loaded in $50 \mathrm{~mL}$ three neck flask and degassed under argon for $20 \mathrm{~min}$ at $100^{\circ} \mathrm{C}$. Then, the temperature of clear brownish manganese precursor solution was increased to $280^{\circ} \mathrm{C}$. At this temperature the solution became transparent; the selenium precursor $(\approx 2 \mathrm{~mL})$ was quickly injected into the main reaction vessel. After the injection, the color of the solution turned faint yellow indicating formation of MnSe nanoclusters. The temperature of the main reaction was dropped to $260^{\circ} \mathrm{C}$ and was held there for $1 \mathrm{~h}$. At the same time, the zinc stock solution was heated at $125^{\circ} \mathrm{C}$ under the argon gas flow until a clear solution was formed. The reaction temperature of main reaction was set to $290^{\circ} \mathrm{C}$ for the injection of zinc precursor. Once it hit the target temperature, $3 \mathrm{~mL}$ of the zinc precursor solution was injected quickly. Immediately after the Zinc precursor injection, the solution glow yellow under UV-light, showing the growth of $\mathrm{ZnSe}$ over MnSe NCs. The temperature of the main reaction was decreased to $260^{\circ} \mathrm{C}$ and held there for $20 \mathrm{~min}$. Amine precursor solution was added to the main reaction. The same injection strategy was performed three times with 20 min intervals for the completion of the reaction and all the zinc precursor was injected into the main reaction. The growth process was monitored through successive UV-Vis and PL measurements. Finally, the reaction was cooled to room temperature. We used a new method for purification of these NCs. For purification, the NCs were warmed and centrifuged. The lower part was taken and added methanol, then the solution was mixed well and warmed again. After taking the lower part and adding toluene, iso-propanol and methanol the mixture is precipitated. Finally, toluene was added and centrifuged. Finally, 
the NCs were dissolved in toluene. The NCs were stable in toluene and showed no sign of aggregation for a long time.

Water-soluble Mn-doped ZnSe NCs: Purified NCs were dissolved in a minimum volume of chloroform and an excess amount of 3-mercaptopropionic acid (MPA) was added until the solutions became cloudy. The mixture was then shaken for a $24 \mathrm{~h}$. The MPA capped NCs were flocculated and then were centrifuged. Finally, desired amount of water was added to the precipitated NCs and very little tetramethylammonium hydroxide solution was added drop wise until all the NCs get transferred to water.

Characterization of Mn-doped ZnSe NCs: UV-Vis spectra were obtained using a UV-Vis spectrophotometer (Varian - Cary 100). Photoluminescence ( $\mathrm{PL}$ ) spectra (both excitation and emission) and time-resolved fluorescence measurements of the NCs were obtained with a fluorescence spectrophotometer (Varian - Cary Eclipse). The quantum yield of the NCs was measured using Horiba Jobin Yvon Time resolved fluorescence setup using an integrating sphere F-3018. Transmission electron microscopy (TEM - Tecnai G2 F30) images were obtained using a high resolution transmission electron microscope (HRTEM) operating at $300 \mathrm{kV}$. Elemental analysis is done using energy dispersive x-ray spectroscopy (EDS), X-ray photoelectron spectroscopy (XPS - Thermo K-Alpha) and inductive coupled plasma mass spectroscopy (ICPMS - Thermo $X$ Series II). Fluorescence Imaging of NCs drop casted on the quartz were obtained using Carl Zeiss Axio Scope Fluorescence Microscope. An excitation wavelength of $405 \mathrm{~nm}$ (LED) (power less than $5 \mathrm{~mW}$ ) was used with a 560-600 nm emission pass filter. Magnetic Resonance imaging experiments were performed at room temperature on a 3 T Siemens TrioTim MRI scanner. MRIs were acquired using a spin echo image sequence (slice thickness $=3 \mathrm{~mm}$, flip angle $=90^{\circ}$, acquisition matrix $=256$ pixels $\times 256$ pixels, FoV $=90 \mathrm{~mm} \times 90 \mathrm{~mm}, \mathrm{TE}=13 \mathrm{~ms}$, TR from 100 to $10000 \mathrm{~ms}$ ), where $T_{1}$ is extracted by fitting an exponential function to these curves by using least squares curve fitting algorithm. The longitudinal $\left(r_{1}\right)$ relaxivity was determined from the following equation: ${ }^{[9]}$

$r_{1}[\mathrm{Mn}]=\frac{1}{T_{1}}-\frac{1}{T_{0}}$

where, $T_{0}$ and $T_{1}$ are longitudinal relaxation times of DI water and samples with increasing $\mathrm{Mn}^{2+}$ concentration.

\section{Supporting Information}

Supporting Information is available from the Wiley Online Library or from the author.

\section{Acknowledgements}

This work is supported by EU-FP7 Nanophotonics4Energy NoE, and TUBITAK EEEAG, 110E217. HVD gratefully acknowledges ESF-EURYI and TUBA-GEBIP. S.G and Y.K. acknowledge TUBITAK fellowship.

[1] M. Bruchez, M. Moronne, P. Gin, S. Weiss, A. P. Alivisatos, Science 1998, 281, 2013-2016.

[2] X. Michalet, F. F. Pinaud, L. a Bentolila Bentolila, J. M. Tsay, S. Doose, J. J. Li, G. Sundaresan, A. M. Wu, S. S. Gambhir, S. Weiss, Science 2005, 307, 538-44.
[3] M.-F. Bellin, Eur. J. Radiol. 2006, 60, 314-23.

[4] Z. Ali, A. Z. Abbasi, F. Zhang, P. Arosio, A. Lascialfari, M. F. Casula, A. Wenk, W. Kreyling, R. Plapper, M. Seidel, R. Niessner, J. Knöll, A. Seubert, W. J. Parak, Anal. Chem. 2011, 83, 2877-82.

[5] V. C. Pierre, M. Botta, K. N. Raymond, J. Am. Chem. Soc. 2005, $127,504-5$.

[6] P. Caravan, J. J. Ellison, T. J. McMurry, R. B. Lauffer, Chem. Rev. 1999, 99, 2293-352.

[7] J. Gao, H. Gu, B. Xu, Acc. Chem. Res. 2009, 42, 1097-107.

[8] J. Kim, Y. Piao, T. Hyeon, Chem. Soc. Rev. 2009, 38, 372-90.

[9] E. A. Weitz, C. Lewandowski, E. D. Smolensky, M. Marjańska, V. C. Pierre, ACS Nano 2013, 7, 5842-5849.

[10] D. Gerion, J. Herberg, R. Bok, E. Gjersing, E. Ramon, R. Maxwell, J. Kurhanewicz, T. F. Budinger, J. W. Gray, M. A. Shuman, F. F. Chen, J. Phys. Chem. C 2007, 111, 12542-12551.

[11] J. Park, S. Bhuniya, H. Lee, Y.-W. Noh, Y. T. Lim, J. H. Jung, K. S. Hong, J. S. Kim, Chem. Commun. 2012, 48, 3218-3220.

[12] J. Kim, J. E. Lee, J. Lee, Y. Jang, S.-W. Kim, K. An, J. H. Yu, T. Hyeon, Angew. Chem. Int. Ed. Ed. 2006, 45, 4789-93.

[13] H.-Y. Xie, C. Zuo, Y. Liu, Z.-L. Zhang, D.-W. Pang, X.-L. Li, J.-P. Gong, C. Dickinson, W. Zhou, Small 2005, 1, 506-9.

[14] T. R. Sathe, A. Agrawal, S. Nie, Anal. Chem. 2006, 78, 5627-32.

[15] D. Koktysh, V. Bright, W. Pham, Nanotechnology 2011, 22, 275606.

[16] T. Jin, Y. Yoshioka, F. Fujii, Y. Komai, J. Seki, A. Seiyama, Chem. Commun. 2008, 0, 5764-5766.

[17] C. Kirchner, T. Liedl, S. Kudera, T. Pellegrino, A. Muñoz Javier, H. E. Gaub, S. Stölzle, N. Fertig, W. J. Parak, Nano Lett. 2005, 5, 331-8.

[18] D. Pan, A. H. Schmieder, S. a Wickline Wickline, G. M. Lanza, Tetrahedron 2011, 67, 8431-8444.

[19] F. G. Shellock, E. Kanal, J. Magn. Reson. Imaging. Imaging 1999, $10,477-484$.

[20] V. M. Runge, J. Magn. Reson. Imaging. Imaging 2000, 12, 205-213.

[21] N. Pradhan, X. Peng, J. Am. Chem. Soc. 2007, 129, 3339-47.

[22] J. H. Yu, S.-H. Kwon, Z. Petrášek, O. K. Park, S. W. Jun, K. Shin, M. Choi, Y. Il Park Park, K. Park, H. Bin Na, N. Lee, D. W. Lee, J. H. Kim, P. Schwille, T. Hyeon, Nat. Mater. 2013, 12, 359-66.

[23] S. Wang, B. R. Jarrett, S. M. Kauzlarich, A. Y. Louie, J. Am. Chem. Soc. 2007, 129, 3848-56.

[24] M. Gaceur, M. Giraud, M. Hemadi, S. Nowak, N. Menguy, J. P. Quisefit, K. David, T. Jahanbin, S. Benderbous, M. Boissière, S. Ammar, J. Nanopart. Res. Res. 2012, 14, 932.

[25] R. Koole, W. J. M. Mulder, M. M. van Schooneveld Schooneveld, G. J. Strijkers, A. Meijerink, K. Nicolay, Nanomed. Nanobiotechnol. 2009, 1, 475-491.

[26] W. Q. Peng, S. C. Qu, G. W. Cong, Z. G. Wang, J. Cryst. Growth 2005, 279, 454-460.

[27] A. Aboulaich, M. Geszke, L. Balan, J. Ghanbaja, G. Medjahdi, R. Schneider, Inorg. Chem. 2010, 49, 10940-8.

[28] W. Park, T. C. Jones, W. Tong, S. Schön, M. Chaichimansour, B. K. Wagner, C. J. Summers, J. Appl. Phys. Phys. 1998, 84, 6852.

[29] A. Ishizumi, E. Jojima, A. Yamamoto, Y. Kanemitsu, J. Phys. Soc. Japan 2008, 77, 053705.

[30] C. R. Brundle, C. A. Evans, S. Wilson, Encyclopedia of Materials Characterization: Surfaces, Interfaces, Thin Films, ButterworthHeinemann, Stoneham, USA 1992.

[31] C. Gan, Y. Zhang, D. Battaglia, X. Peng, M. Xiao, Appl. Phys. Lett. 2008, 92, 241111.

[32] D. H. Gultekin, T. E. Raidy, J. C. Gore, Contrast Media Mol. Imaging 2009, 4, 33-36.

Received: April 25, 2014 Revised: June 13, 2014

Published online: August 8, 2014 